\title{
An Exact Close-form PEP and a new PEP for Space-Time codes
}

\author{
Z. Zhang, S.W.Cheung and T.I.Yuk \\ Department of Electronic \& Electrical Engineering, University of Hong Kong, Pokfulam Road, Hong Kong
}

\begin{abstract}
A close-form expression of the exact Pair-wise Error Probability (PEP) for Space-Time (S-T) codes in Rayleigh fading channel is derived using the probabilitydensity function (PDF) of a sum of independent exponential distributed random variables. Since the expression requires evaluating the coefficients for partial fraction expansion, an easy analytical way is proposed for doing this. The exact PEP is subsequently used to develop a simple PEP using upper bound. Both PEPs are used for error rate evaluation. Numerical calculations and Monte Carlo computer simulation have been used to study the accuracies of these PEPs for error rate evaluation of a rotation-based diagonal S$T$ code (D code) in Rayleigh fading channels. Four other PEPs based on different bounds are also studied for comparison. Results show that our derived close-form PEP is an exact $P E P$ and our proposed $P E P$ is a very tight bound to the exact PEP.
\end{abstract}

Index Terms- PEP, Space-Time codes

\section{INTRODUCTION}

Accurate Pair-wise Error Probability (PEP) is important for the construction of S-T codes [1] and PEP is a wellknown tool for deriving the error rates for other coding schemes [2]. However, the exact PEP formulas developed so far involve either numerical approximations [3] or the residue [4] which is not in close-form, or $M^{\text {th }}$ order derivative [5 6] which is not easy to do in an analytical way if $M$ is large. A different approach is presented in this paper. We first derive a general and exact close-form PDF of a sum of independent exponential distributed random variables and then use it to develop an exact close-form PEP. From these results, we propose a new PEP based on the upper bound. Same as other exact close-form PEPs [5 6], the proposed PEP expressions also requires evaluation of the coefficients for partial fraction expansion. However, we propose an easy way to do this, avoiding the calculation of high order derivatives. The complexity analysis shows that the proposed method has much less computation load than the method using high order derivatives.

Numerical calculations and Monte Carlo computer simulations are used to assess the accuracies of the exact PEP and the proposed PEP for bit-error-rate (BER) evaluation of a rotation-based diagonal S-T code ( $D$ code) in a block Rayleigh fading channel. For comparison, four other PEPs, i.e., the PEPs based on the Chernoff bound [1], the Asymptotic bound [1], the tight Asymptotic bound [7] and the Eigen-Geometric-Mean (EGM) bound [8], are also studied for comparison. Results show that the exact closeform PEP derived in this paper is a true exact PEP and our proposed PEP is much tighter than the other four PEPs studied.

The remainder of this paper is organized as follows. Section II describes the system model. The exact closeform PEP is derived in section III. In section IV, our proposed PEP is derived and the other four PEPs are also introduced. Results and discussions are reported in section V. Finally, conclusions are drawn in section VI.

\section{SYSTEM MODEL}

The S-T coded system considered here is modeled as:

$$
\mathbf{R}=\mathbf{H X}+\mathbf{Y}
$$

where $\mathbf{H}$ is a $M \times N$ channel matrix with $N$ and $M$ being the number of transmit and receive antennas, respectively. Each of the elements $\left\{h_{m, n}\right\}$ in $\mathbf{H}$ is the channel transfer function from the $n$-th transmit antenna to the $m$-th receive antenna and modeled as independent and identically distributed (i.i.d) Complex Gaussian random variable (RV) with zero mean and variance 0.5 for both real and imaginary parts. In (1), $\mathbf{X}$ is a $N \times L$ matrix where $L$ is the number of time intervals in a block. Each of the elements $\left\{x_{n, t}\right\}$ in $\mathbf{X}$ is a coded symbol transmitted from the $n$-th transmit antenna in the $t$-th time interval with average bit energy $E_{b}$. The received signal matrix $\mathbf{R}$ is a $M \times L$ matrix with each of elements in $\left\{r_{m, t}\right\}$ being the element received from the $m$-th antenna in the $t$-th time interval. Additive white Gaussian noise (AWGN) in the channel is modeled by a $M \times L$ matrix $Y$ with all elements being i.i.d complex Gaussian random variables with zero-mean and variance $N_{0} / 2$ for both real and imaginary parts. Maximum-Likelihood (ML) detection is assumed to be used at the receiver. 


\section{EXACT CLOSE-FORM PEP FOR S-T CODES}

3.1 Conditional PEP:

The conditional PEP of two different codewords $\mathbf{X}$ and $\hat{\mathbf{X}}$, on a $\mathbf{H}$, is [9]:

$P_{e}(\mathbf{X} \rightarrow \hat{\mathbf{X}} \mid \mathbf{H})=Q\left(\sqrt{d^{2}(\mathbf{X}, \hat{\mathbf{X}}) S N R / 2}\right)$

where $S N R=E_{b} / N_{0}$ and:

$d^{2}(\mathbf{X}, \hat{\mathbf{X}})=\sum_{m=1}^{M} \sum_{n=1}^{N} \lambda_{n}\left|\beta_{n, m}\right|^{2}$

is referred in [1] as the modified Euclidean distance between the two codewords. In (3), $\lambda_{n}$ is the $n$-th eigenvalue of the matrix $(\mathbf{X}-\hat{\mathbf{X}})(\mathbf{X}-\hat{\mathbf{X}})^{*}$ (where $*$ is denoted as the transpose conjugate). $\beta_{n, m}$ in (3) is an i.i.d. complex Gaussian RV with zero mean [1], so $d^{2}(\mathbf{X}, \hat{\mathbf{X}})$ is a sum of independent exponential random variables $\left\{\lambda_{n}\left|\beta_{n, m}\right|^{2}\right\}$ with $\left\{\lambda_{n}\right\}$ being the means. If all $\left\{\lambda_{n}\right\}$ are equal, $d^{2}(\mathbf{X}, \hat{\mathbf{X}})$ is a central Chi-square distributed random variable and the PDF has been derived in [10]. If all $\left\{\lambda_{n}\right\}$ are distinct, the PDF has also been derived in [10]. If some of $\left\{\lambda_{n}\right\}$ are identical and the rest are all distinct, then the PDF has been derived in [11]. However, to our knowledge, the PDF of $d^{2}(\mathbf{X}, \hat{\mathbf{X}})$ in a general case of $\left\{\lambda_{n}\right\}$ has not been studied. In the next section, we consider this general case.

\subsection{PDF of the modified Euclidean distance:}

We only consider the unequal case here. Suppose there are $K$ non-zero eigenvalues, among them there are $R$ distinct eigenvalues, i.e., $\left\{\begin{array}{llllll}\tilde{\lambda}_{1} & \ldots & \tilde{\lambda}_{r} & \ldots & \tilde{\lambda}_{R}\end{array}\right\}$. Denoting $W_{r}$, for $r=1, \ldots, R$, as the number of eigenvalues having the same value $\tilde{\lambda}_{r}$ and using partial-fraction expansion, the characteristic function $(\mathrm{CF})$ of $d^{2}(\mathbf{X}, \hat{\mathbf{X}})$ can be written as:

$$
\varphi_{d}(j \omega)=\prod_{r=1}^{R}\left(1-j \omega \tilde{\lambda}_{r}\right)^{-M W_{r}}=\sum_{r=1}^{R} \sum_{s=1}^{M W_{r}} A_{r, s}\left(1-j \omega \tilde{\lambda}_{r}\right)^{-s}
$$

where $j=\sqrt{-1}, \omega$ is the frequency and $A_{r, s}$ is determined by [12] :

$A_{r, s}=\frac{(-1)^{M W_{r}-s}}{\left(\tilde{\lambda}_{r}\right)^{M W_{r}-s}\left(M W_{r}-s\right) !}$

$\left.\left\{\frac{d^{M W_{r}-s}}{d(j \omega)^{M W_{r}-s}}\left[\prod_{i=1, i \neq r}^{R} \frac{1}{\left(1-j \omega \tilde{\lambda}_{i}\right)^{M W_{i}}}\right]\right\}\right|_{j \omega=\frac{1}{\tilde{\lambda}_{r}}}$

Performing the inverse Fourier transform (IFT) on (4) yields the expected PDF:
$p_{d}(x)=\sum_{r=1}^{R} \sum_{s=1}^{M W_{r}-s} A_{r, s}\left(\frac{1}{(s-1) !\left(\tilde{\lambda}_{r}\right)^{s}} x^{(s-1)} e^{-\frac{x}{\tilde{\lambda}_{r}}}\right)$

which shows that $p_{d}(x)$ could be evaluated in close-form. However, evaluating the coefficient $A_{r, s}$ using (5) involves high order derivatives on a product of different algebraic functions. When there are more than two distinct eigenvalues $(R>2)$ and $M W_{r}$ is large, it becomes impractical to evaluate these high order derivatives analytically. In the next section, we introduce a simple way to determine $A_{r, s}$.

\subsection{Determination of $A_{r, s}$}

It can be readily shown that:

$$
\begin{aligned}
& A_{r, s}=(-1)^{M W_{r}-s} \prod_{i=1, i \neq r}^{R}\left(\frac{\tilde{\lambda}_{r}}{\widetilde{\lambda}_{r}-\tilde{\lambda}_{i}}\right)^{M W_{i}} \\
& \left\{\sum_{\substack{t_{1}, \ldots, t_{i}, \ldots, t_{R}, i \neq r \\
t_{i} \geq 0, \sum_{i=1, i \neq r}^{R} t_{i}=M W_{r}-s}}\left[\prod_{i=1, i \neq r}^{R}\left(\frac{\tilde{\lambda}_{i}}{\tilde{\lambda}_{r}-\tilde{\lambda}_{i}}\right)^{t_{i}}\left(\begin{array}{c}
t_{i}+M W_{i}-1 \\
M W_{i}-1
\end{array}\right)\right]\right\}
\end{aligned}
$$

where the summation before the square bracket is to sum up the products of combinations for non-negative integers $t_{1}, \ldots ., t_{i}, \ldots t_{R} \quad$ (excluding $t_{r}$ ) with the constraint $\sum_{i=1, i \neq r}^{R} t_{i}=M W_{r}-s$

Computation advantages of using (7):

Here we discuss the complexities of using (5) and (7) to compute $A_{r, s}$. Consider a simple case with $R=M$ and $W_{r}=1$. To compute $A_{r, s}$ using (7), most of the operations are the computations of the products inside the square bracket. Each of these products is composed of $R-1$ different factors, so the complexity of using (7) to compute $A_{r, s}$ is proportional to the number of these $(R-1)$-factor products required to be evaluated. While if (5) is used, a high-order-derivative operation is required to be performed on a product composing of $R-1$ different factors, each having $M^{\text {th }}$ order. From the product rule of derivatives, the high-order-derivative operation in (5) can be expanded into a number of products composing of $(R-1)$ factors, each having a derivative operation. The complexity of using (5) to compute $A_{r, s}$ is therefore also proportional to the number of these $(R-1)$-factor products required to be evaluated. However, each of these products here has a derivative factor and so should be more difficult to evaluate. For convenience, we assume that the $(R-1)$-factor products in both methods require about the same 
complexity to evaluate, then we can assess their complexities by evaluating the numbers of $(R-1)$-factor products required to be evaluated in using these two methods. For $M=R=3$ to 7 and $s=1$, the numbers of $(R-1)$ factor products required to evaluate $A_{r, s}$ using (7) and (5) are showed in Table 1 . The advantage of using (7) is very obvious as $M$ becomes large.

\begin{tabular}{|c|c|c|}
\hline$M$ & Using (7) & Using (5) \\
\hline 3 & 3 & 6 \\
\hline 4 & 10 & 39 \\
\hline 5 & 35 & 340 \\
\hline 6 & 126 & 3905 \\
\hline 7 & 468 & 55986 \\
\hline
\end{tabular}

Table 1: Numbers of $(R-1)$-factor products required to determine $A_{1,1}$ for $M=R=3 \sim 7$ and $s=1$ using (7) and (5)

\subsection{Exact close-form PEP:}

The exact PEP can be obtained by averaging the conditional PEP over $p_{d}(x)$. Using the exact PEP of the equal-eigenvalue case in [10], the exact PEP of the unequal-eigenvalue case can be obtained as:

$$
\begin{aligned}
& P_{e}(\mathbf{X} \rightarrow \hat{\mathbf{X}})=\sum_{r=1}^{R} \sum_{s=1}^{M W_{r}} A_{r, s}\left(\left[0.5\left(1-u_{r}\right)\right]^{s} \sum_{k=0}^{s-1}\left[\left(\begin{array}{c}
s-1+k \\
k
\end{array}\right)\left(0.5\left(1+u_{r}\right)\right)^{k}\right]\right), \\
& u_{r}=\sqrt{\frac{\tilde{\lambda}_{r} S N R}{4+\tilde{\lambda}_{r} S N R}}
\end{aligned}
$$

which was first reported in [5] where $A_{r, s}$ is determined by calculating the high order derivatives as shown in (5) and sometimes hard to do. However, $A_{r, s}$ can be determined using (7), avoiding the calculations of high order derivatives. It should also be noted that the PDF of $d^{2}(\mathbf{X}, \hat{\mathbf{X}})$ is a useful tool in communications systems. For example, it can be used to derive the outage probability for repetition codes [11], and the BER of RAKE receiver [10]. Since the close-form PDF of the general case remains an open problem, the authors attempt to solve it here.

\section{PEPS BASED ON DIFFERENT UPPER BOUNDS}

\subsection{Proposed PEP}

To evaluate the exact close-form PEP, it requires to carry out the product of three summation functions as shown in (8). Here we propose a relatively simpler PEP based on the upper bound for the $Q$ function [13]:

$$
Q(x)<\frac{1}{\sqrt{2 \pi}} \frac{e^{-\frac{x^{2}}{2}}}{x}, \quad x>0
$$

\section{Equal-eigenvalue case}

Using (9), a new PEP for the equal-eigenvalue case is:

$$
P_{e}(\mathbf{X} \rightarrow \hat{\mathbf{X}})<\frac{2}{u}\left(\frac{1-u^{2}}{4}\right)^{M K}\left(\begin{array}{c}
2 M K-2 \\
M K-1
\end{array}\right), u=\sqrt{\frac{\lambda S N R}{4+\lambda S N R}}
$$

\section{Unequal eigenvalue case}

Similarly, the new PEP for the unequal-eigenvalue case can be obtained as:

$$
\begin{aligned}
& P_{e}(\mathbf{X} \rightarrow \hat{\mathbf{X}})<\sum_{r=1}^{R} \sum_{s=1}^{M W_{r}} A_{r, s} \frac{2}{u_{r}}\left(\frac{1-u_{r}^{2}}{4}\right)^{s}\left(\begin{array}{c}
2 s-2 \\
s-1
\end{array}\right) \\
& u_{r}=\sqrt{\frac{\tilde{\lambda}_{r} S N R}{4+\tilde{\lambda}_{r} S N R}}
\end{aligned}
$$

(10) and (11) are our proposed PEPs for the equal- and unequal- eigenvalue cases, respectively. Comparing with (8), it can be seen that (11) has a product of only two summation functions and so is relatively simpler to use.

\subsection{Four PEPs studied}

In order to compare the accuracy of our proposed PEP, four PEPs based on the Chernoff bound, the Asymptotic bound, the Tight Asymptotic bound and the EigenGeometric-Mean (EGM) bound, are also studied and briefly described here.

\section{PEP based on Chernoff bound}

Using the Chernoff bound for the $Q$ function [13], the PEP based on the Chernoff bound is [1]:

$$
P_{e}(\mathbf{X} \rightarrow \hat{\mathbf{X}}) \leq\left(\prod_{i=1}^{K}\left(1+\lambda_{i} S N R / 4\right)\right)^{-M}
$$

\section{PEP based on Asymptotic bound}

At high SNRs, (12) can be further bounded by an Asymptotic bound [1]:

$$
P_{e}(\mathbf{X} \rightarrow \hat{\mathbf{X}}) \leq\left(\prod_{i=1}^{K} \lambda_{i}\right)^{-M}(S N R / 4)^{-K M}
$$

\section{PEP based on Tight Asymptotic bound}

A PEP based on the tight asymptotic bound was proposed in [7] as:

$$
P_{e}(\mathbf{X} \rightarrow \hat{\mathbf{X}}) \leq \frac{1}{2}\left(\begin{array}{c}
2 K M \\
K M
\end{array}\right)\left(\prod_{i=1}^{K} \lambda_{i}\right)^{-M}(S N R)^{-K M}
$$

PEP based on Eigen-Geometric-Mean (EGM) bound In [8], the geometric-mean of eigenvalues was used to develop a PEP: 


$$
\begin{aligned}
& P_{e}(\mathbf{X} \rightarrow \hat{\mathbf{X}}) \leq[0.5(1-\bar{u})]^{M K} \sum_{k=0}^{M K-1}\left[\left(\begin{array}{c}
M K-1+k \\
k
\end{array}\right)(0.5(1+\bar{u}))^{k}\right] \\
& \text { where } \bar{u}=\sqrt{\frac{\bar{\lambda} S N R}{4+\bar{\lambda} S N R}} \text { and } \bar{\lambda}=\left(\prod_{i=1}^{K} \lambda_{i}\right)^{\frac{1}{K}}
\end{aligned}
$$

\section{RESULTS AND DISCUSSIONS}

\subsection{Rotation based $S$-T code and Union bound}

For 2 by 2 rotation code construction, four binary independent information symbols, $s_{1,1}, s_{1,2}, s_{2,1}, s_{2,2}$ are coded and placed in a diagonal [14] way to form a code matrix $\mathbf{X}$ :

$$
\mathbf{X}=\left[\begin{array}{cc}
\cos \left(\theta_{1}\right) s_{1,1}-\sin \left(\theta_{1}\right) s_{1,2} & \sin \left(\theta_{2}\right) s_{2,1}+\cos \left(\theta_{2}\right) s_{2,2} \\
\cos \left(\theta_{2}\right) s_{2,1}-\sin \left(\theta_{2}\right) s_{2,2} & \sin \left(\theta_{1}\right) s_{1,1}+\cos \left(\theta_{1}\right) s_{1,2}
\end{array}\right]
$$

Since the operations of (17) can be thought of as rotating the column vectors by the corresponding angles and the rotated (or coded) signal elements are transmitted in a diagonal way, we call such code a rotation-based diagonal space-time code (D code). For simplicity, BPSK is used for study.

For comparison, the Union bound for the BER has been used to study the accuracy of our proposed PEP and other PEPs and is given by [2]:

$$
P_{U}=\frac{1}{C} \sum_{\mathbf{X}} \sum_{\hat{\mathbf{x}} \mid \hat{\mathbf{X}} \neq \mathbf{X}} \frac{e(\mathbf{X}, \hat{\mathbf{X}})}{B} P_{e}(\mathbf{X} \rightarrow \hat{\mathbf{X}})
$$

where $e(\mathbf{X}, \hat{\mathbf{X}})$ is the number of bits in error due to the error event $(\mathbf{X} \rightarrow \hat{\mathbf{X}}), B$ is the number of bits per codeword and $C$ is the size of the codebook. In (18), all codewords are assumed equally likely to be transmitted.

\subsection{Results on D-code}

A series of Monte Carlo simulations have been used to evaluate the BERs of the D-code system using an optimum angle pair $(1.368,0.531)$ obtained in [15] with 2 receive and 2 transmit antennas. The numerical calculations on the BERs of the same system using the proposed PEPs of (10) and (11) have also been carried out and results are shown in Fig. 1. For comparison, the results of the numerical calculations using different PEPs, i.e., the exact close-form PEPs of (8), and the PEPs of (12) (15) based on the Chernoff bound, the Asymptotic bound, the Tight Asymptotic bound, and the EGM bound, respectively, are all shown in Fig. 1. It can be seen that the exact close-form PEP produces results very close to simulations, especially at high SNRs, and thus is very close to the true PEP. At $\mathrm{BER}=10^{-3}$, it differs by about $0.25 \mathrm{~dB}$ from the simulation result. Our proposed PEP is quite close to the exact closed-form PEP and, at BER $=10^{-3}$, it differs by about $0.5 \mathrm{~dB}$ from the simulation result. In fact, in most SNRs tested, the differences between the results of the exact PEP and our proposed PEP are less than $0.3 \mathrm{~dB}$, indicating that our proposed PEP is quite close to the exact PEP. Fig. 1 shows that the PEP based on the EGM bound is the best one among the other PEPs studied. At BER = $10^{-3}$, it differs from the simulation results by about $2 \mathrm{~dB}$. The PEP based on the tight Asymptotic bound is less accurate than the PEP based on the Chernoff bound at low SNRs, but is better at high SNR. At BER $=10^{-3}$, the PEPs based on the tight Asymptotic bound and the Chernoff bound have differences of about $3 \mathrm{~dB}$ and $3.2 \mathrm{~dB}$ from the simulation result and so they are just loose bounds only. The PEP based on the Asymptotic bound is the worst one, having the largest difference from the simulation. At BER $=10^{-3}$, the difference is about $5 \mathrm{~dB}$. Clearly, our proposed PEP is superior to all other PEPs studied.

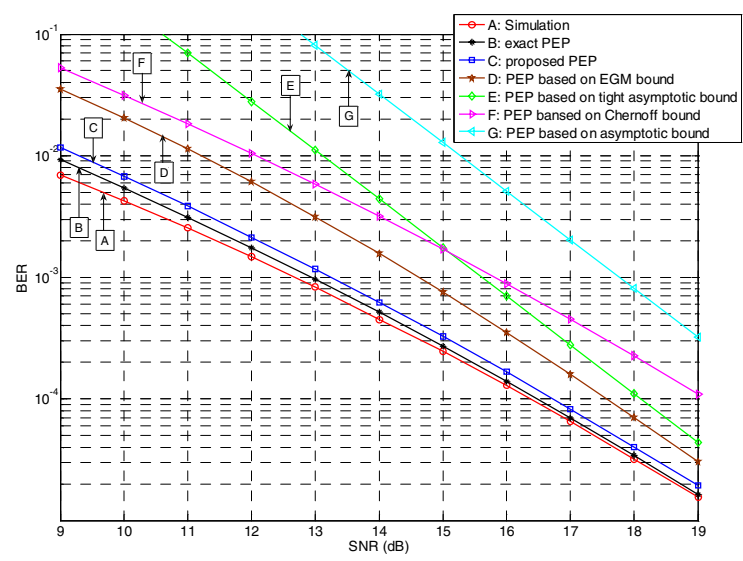

Fig 1 BER of D code using different Union bounds and 2 receive antennas

\section{ConCLusions}

An exact close-form PEP has been derived using the PDF of a sum of independent exponential distributed random variables. A method requiring much less computation complexity than the method using high order derivative has been studied to compute the coefficients for partial fraction expansion. A simple PEP developed from the exact close-form PEP has been proposed. Numerical analysis and Monte Carlo computer simulation have been used to study the exact close-form PEP and the proposed PEP, together with other PEPs, in a S-T coded system in independent Rayleigh fading channels. Results have shown that our close-form PEP formula is an exact PEP and the proposed PEP is a very tight bound to the exact PEP. 


\section{REFERENCES}

[1]. V. Tarokh, N. Seshadri, and A. R. Calderbank, "Space-time codes for high data rate wireless communication: Performance criterion and code construction," IEEE Trans on Information Theory, vol. 44, pp. 744-765, Mar.1998.

[2]. S. Benedetto, E.Biglieri, "Principles of Digital Transmission with wireless applications", kluwer, New York, 1999

[3]. G. Taricco and E. Biglieri, "Exact pairwise error probability of space-time codes," IEEE Trans. Inform. Theory, pp. 510-513, no.2, vol. 48, Feb. 2002

[4]. M. Uysal, C.N. Georghiades, "On the error performance analysis of space-time trellis codes", Wireless Communications, IEEE Trans on, pp:1118 - 1123, no.4, vol.3, July 2004

[5] H.F. Lu, Y.K Wang, P.V. Kumar and K.M. Chugg, "Remarks on space-time codes including a new lower bound and an improved code", IEEE Trans. Information Theory, pp:2752 - 2757 no.10, vol.49, Oct. 2003

[6] M. K. Simon and M.S. Alouini, "Digital Communication Over Fading Channels", 2nd ed. New York: Wiley, 2005

[7] M.P. Fitz, J. Grimm and S. Siwamogsatham, "A new view of performance analysis techniques in correlated Rayleigh fading", IEEE WCNC 99, pp. 139-144, vol.1, Sept. 1999

[8] M-K Byun and B.G. Lee, "New bounds of pairwise error probability for space-time codes in Rayleigh fading channels", IEEE WCNC 02, pp.89 - 93, vol.1, March 2002

[9] B.Vucetic and J.H.Yuan, "Space-time coding", John Wiley, 200B.Vucetic and J.H.Yuan, "Space-time coding", John Wiley, 2003

[10] J.G. Proakis, "Digital Communications", $4^{\text {th }}$ Edition, McGraw-Hill, 2001, Boston, U.S.A,

[11]. H.V. Khuong and H.Y. Kong, "General Expression for pdf of a Sum of Independent Exponential Random Variables", IEEE Communications Letters, pp 159 161, March 2006

[12]. A.V. Oppenheim and A. S. Willsky, "Signals and systems", Englewood Cliffs: Prentice-Hall, 1983

[13]. S.G. Wilson, "Digital modulation and coding", NJ : Prentice Hall, 1996.
[14]. H. Yao and G.Wornell, "Achieving the full MIMO diversity-multiplexing frontier with rotation-based spacetime codes", Annual Allerton Conference on Communication, Control and Computing, Monticello IL, 2003

[15]. Z. Zhang, S.W. Cheung, T.I. Yuk and H. Kuo, "Union bounds for BER Evaluation and Code Optimization for Space-Time codes in 2-by-2 MIMO systems", 2006 IEEE Spring Vehicular Technology Conference, pp. 1506-1510, vol.3, May 2006 